\title{
Convergence of holomorphic chains
}

\author{
by SŁaWOmir Rams (Kraków)
}

\begin{abstract}
We endow the module of analytic $p$-chains with the structure of a secondcountable metrizable topological space.

1. Introduction. A holomorphic p-chain in an open subset $\Omega$ of $\mathbb{C}^{n}$ is a formal locally finite sum $Z=\sum_{j \in J} k_{j} Z_{j}$ where $Z_{j}$ are pairwise distinct irreducible analytic subsets of $\Omega$ of pure dimension $p$ and $k_{j} \in \mathbb{Z} \backslash\{0\}$ for $j \in J$. The set $\bigcup_{j \in J} Z_{j}$ is called the support of the chain $\mathrm{Z}$ and denoted by $|Z|$. Each $Z_{j}$ is called a component of $Z$ and the number $k_{j}$ is the multiplicity of $Z_{j}$.

A holomorphic $p$-chain $\mathrm{Z}$ is positive if the multiplicities of all its components are positive. $\mathcal{G}_{+}^{p}(\Omega)$ denotes the set of positive $p$-chains in $\Omega$. The set of holomorphic $p$-chains in $\Omega$ is endowed with the structure of a free $\mathbb{Z}$-module. We denote it by $\mathcal{G}^{p}(\Omega)$.

Given a 0 -chain and an open relatively compact subset $U$ of $\Omega$ the total multiplicity of $Z$ in $U$ is defined as the sum of multiplicities of all its components contained in $U$. We denote the total multiplicity by $\operatorname{deg}_{U} Z$. When $J$ is finite we extend this definition putting $\operatorname{deg} Z=\sum_{j \in J} k_{j}$.

One can define convergence of chains as the classical weak convergence of the associated currents (for details see e.g. [Ch, §14.1-2]). An attempt to explain the geometrical meaning of this convergence is made in [Ch].

In $[\mathrm{Ch}, \S 12.2]$ the author proves that proper intersection is sequentially continuous and also states that this operation is continuous [Ch, §12.4]. However, he neither defines a topology nor proves the equivalence of sequential continuity and continuity.

The main aim of this note is to define a topology on $\mathcal{G}^{p}(\Omega)$ and to study some properties of this topological space. We shall prove that the result of this construction is second-countable, metrizable, and convergence in it coincides with the one defined in [Ch, $\S 12.2]$.
\end{abstract}

1991 Mathematics Subject Classification: 32B15, 32C25, 32C30, 32C99.

Key words and phrases: holomorphic chains, currents, convergence of chains. 
The topology constructed here is useful in studying the intersections of analytic sets (see $[\mathrm{Tw}],[\mathrm{R}]$ ).

2. Topology of $p$-chains. Let $0 \leq p<n$ be integers, $\Omega$ be an open subset of $\mathbb{C}^{n}$. We shall use the following notation:

- $E=\{z \in \mathbb{C}:|z|<1\}$,

- $\Lambda(n, p)=\{\lambda:\{1, \ldots, p\} \rightarrow\{1, \ldots, n\}: \lambda(1)<\ldots<\lambda(p)\}$,

- $e_{1}, \ldots, e_{n}$ - the canonical basis of $\mathbb{C}^{n}$,

- $\pi_{\lambda}:\left(z_{1}, \ldots, z_{n}\right) \rightarrow\left(z_{\lambda(1)}, \ldots, z_{\lambda(p)}\right), \pi=\pi_{(1, \ldots, p)} \mid E^{n}$,

- $\mathcal{A}(\Omega)=\left\{f: \mathbb{C}^{n} \rightarrow \mathbb{C}^{n}: f\right.$ an affine isomorphism, $\left.f\left(\overline{E^{n}}\right) \subset \Omega\right\}$,

- $\mu(h)$-order of a finite branched holomorphic covering $h$,

- for $Z=\sum k_{j} Z_{j}, z \in Z_{s} \backslash \bigcup_{j \neq s} Z_{j}, m(z, Z)=k_{s}$.

Suppose that $\Omega_{1}, \Omega_{2}$ are open subsets of $\mathbb{C}^{n}$ and $\Omega \subset \Omega_{2}$. Given a biholomorphic mapping $f: \Omega_{1} \rightarrow \Omega_{2}$ and $Z=\sum_{j \in J} k_{j} Z_{j}$ belonging to $\mathcal{G}^{p}(\Omega)$, a new $p$-cycle in $f^{-1}(\Omega)$ can be defined by $f^{*}(Z)=\sum_{j \in J} k_{j} f^{-1}\left(Z_{j}\right)$.

Definition 2.1. Let $V$ be an open subset of $\mathbb{C}^{n}$ containing $\overline{E^{n}}$, and $Z \in \mathcal{G}^{p}(V), Z=\sum_{j \in J} k_{j} Z_{j}$, such that $|Z| \cap\left(\overline{E^{p}} \times \partial E^{n-p}\right)=\emptyset$. Define

$$
\mu(Z)=\sum_{j \in J} k_{j} \mu\left(\pi \mid Z_{j} \cap E^{n}\right) .
$$

Definition 2.2. Let $f_{j} \in \mathcal{A}(\Omega), c_{j} \in \mathbb{Z}$ for $j=1, \ldots, m$ and let $K$ be a compact subset of $\Omega$. Define $U\left(\left\{\left(f_{1}, c_{1}\right), \ldots,\left(f_{m}, c_{m}\right)\right\}, K\right)$ to be the set of all $p$-chains $\mathrm{Z}$ in $\Omega$ such that $|Z| \cap K=\emptyset$ and

$$
|Z| \cap f_{j}\left(\overline{E^{p}} \times \partial E^{n-p}\right)=\emptyset, \quad \mu\left(f_{j}^{*}(Z)\right)=c_{j} \quad \text { for } \quad j=1, \ldots, m .
$$

It is easy to verify the following

Proposition 2.3. If $\Omega$ is an open subset of $\mathbb{C}^{n}$, then in $\mathcal{G}^{p}(\Omega)$ the family $\mathcal{U}(\Omega)=\{U(A, K): A$ is a finite subset of $\mathcal{A}(\Omega) \times \mathbb{Z}, K$ is compact in $\Omega\}$, is a base of a topology.

Definition 2.4. The topology of p-chains in $\Omega$ is defined to be the topology generated by $\mathcal{U}(\Omega)$.

The next proposition is an immediate consequence of the last definition.

Proposition 2.5. Let $Z, Z^{\nu}, \widetilde{Z}^{\nu}, \widetilde{Z} \in \mathcal{G}^{p}(\Omega)$.

1. If $Z^{\nu} \rightarrow Z, \widetilde{Z}^{\nu} \rightarrow \widetilde{Z}$, and $|Z+\widetilde{Z}|=|Z| \cup|\widetilde{Z}|$, then $Z^{\nu}+\widetilde{Z}^{\nu} \rightarrow \widetilde{Z}+Z$.

2. If $Z^{\nu} \rightarrow Z, a \in \mathbb{Z}$, then $a \cdot Z^{\nu} \rightarrow a \cdot Z$.

3. $\sum_{\nu=0}^{\infty} Z^{\nu}$ is convergent iff $Z^{\nu} \rightarrow 0$.

4. If $f$ is an affine isomorphism, then $\mathcal{G}^{p}(\Omega) \ni Z \mapsto f^{*}(Z) \in \mathcal{G}^{p}\left(f^{-1}(\Omega)\right)$ is a homeomorphism. 
EXAmple 2.6. $\Omega=\mathbb{C}^{2}, Z^{\nu}=\{1 / \nu\} \times \mathbb{C}, Z=\{0\} \times \mathbb{C}, \widetilde{Z}^{\nu}=(-1)$. $(\{-1 / \nu\} \times \mathbb{C}), \widetilde{Z}=(-1) \cdot(\{0\} \times \mathbb{C})$. Then $Z^{\nu} \rightarrow Z$ and $\widetilde{Z}^{\nu} \rightarrow \widetilde{Z}$ but $Z^{\nu}+\widetilde{Z}^{\nu}$ does not converge to $\widetilde{Z}+Z$. Hence addition is not continuous on $\mathcal{G}^{p}(\Omega)$. Proposition 2.5.1 and Theorem 2.9 give its continuity on $\mathcal{G}_{+}^{p}(\Omega)$.

Given $Z^{1}, \ldots, Z^{k}$ belonging to $\mathcal{G}^{p_{1}}(\Omega), \ldots, \mathcal{G}^{p_{k}}(\Omega)$, respectively, and satisfying the conditions

1. the sum of the codimensions of $\left|Z^{j}\right|$ is equal to $n$,

2. $\bigcap_{j=1}^{k}\left|Z^{j}\right|$ is zero-dimensional,

a 0 -chain is defined by

$$
Z^{1} \wedge \ldots \wedge Z^{k}=\sum_{a \in\left|Z^{1}\right| \cap \ldots \cap\left|Z^{k}\right|} i\left(Z^{1} \wedge \ldots \wedge Z^{k}, a\right) \cdot\{a\}
$$

where $i\left(Z^{1} \wedge \ldots \wedge Z^{k}, a\right)$ denotes the intersection multiplicity defined in [Dr] (see also $[\mathrm{Ch}]$ ). It is easy to prove that in Definition 2.1,

$$
\mu(Z)=\operatorname{deg}_{E^{n}}\left(\left(\{w\} \times E^{n-p}\right) \wedge Z\right) \text { for } w \in E^{p} .
$$

If $f: \Omega_{1} \rightarrow \Omega_{2} \supset \Omega$ is biholomorphic, then by [Ch, $\S 12.3$ ],

$$
i\left(Z^{1} \wedge \ldots \wedge Z^{k}, f(a)\right)=i\left(f^{*}\left(Z^{1}\right) \wedge \ldots \wedge f^{*}\left(Z^{k}\right), a\right) .
$$

Proposition 2.7. Let $Z^{\nu}, Z \in \mathcal{G}^{p}(\Omega)$. If for each compact $K \subset \Omega \backslash|Z|$ we have $\left|Z^{\nu}\right| \cap K=\emptyset$ for almost all $\nu$, then the following conditions are equivalent:

1. For each point $a \in \operatorname{Reg}|Z|$, each $(n-p)$-dimensional plane transversal to $|Z|$ at $a$ and each open set $U$ relatively compact in $L$ such that $\bar{U} \cap|Z|=$ $\{a\}$ there is an index $\nu_{0}$ such that $\operatorname{dim}\left(\left|Z^{\nu}\right| \cap U\right)=0, \operatorname{deg}_{U}\left(Z^{\nu} \wedge L\right)=$ $\operatorname{deg}_{U}(Z \wedge L)$ for all $\nu>\nu_{0}$.

2. For each point a from a given dense subset $D \subset \operatorname{Reg}|Z|$, each $(n-p)$ dimensional plane transversal to $|Z|$ at $a$ and each open set $U$ relatively compact in $L$ such that $\bar{U} \cap|Z|=\{a\}$ there is an index $\nu_{0}$ such that $\operatorname{dim}\left(\left|Z^{\nu}\right| \cap U\right)=0, \operatorname{deg}_{U}\left(Z^{\nu} \wedge L\right)=\operatorname{deg}_{U}(Z \wedge L)$ for all $\nu>\nu_{0}$.

3. $Z^{\nu} \rightarrow Z$ in the topology of p-chains.

Proof. The proposition is obvious for $p=0$ or $Z=0$. Let $p>0$, $Z \neq 0$.

$1 \Rightarrow 2$. Obvious.

$2 \Rightarrow 3$. Let $Z \in U(A, K), A=\left\{\left(f_{1}, c_{1}\right), \ldots,\left(f_{m}, c_{m}\right)\right\}$. We check that $Z^{\nu} \in$ $U(A, K)$ for sufficiently large $\nu$. Since $U(A, K)=\bigcap_{j=1}^{m} U\left(\left\{\left(f_{j}, c_{j}\right)\right\}, K\right)$ we can assume $m=1$. By Proposition 2.5.4 it suffices to consider $f_{1}=\operatorname{id}_{\mathbb{C}^{n}}$. Fix $w \in E^{p}$ such that $\{w\} \times E^{n-p}$ is transversal to $|Z|$ at each point of the set $\left(\{w\} \times E^{n-p}\right) \cap|Z|=\left\{z_{1}, \ldots, z_{s}\right\}$. There exist $\varepsilon>0$ and open pairwise disjoint relatively compact subsets $U_{1}, \ldots, U_{s}$ of $E^{n-p}$ such that: 
- $w+\varepsilon \overline{E^{p}} \subset E^{p}$

- $|Z| \cap\left(\{w\} \times \bar{U}_{j}\right)=\left\{z_{j}\right\}$ for $j=1, \ldots, s$,

- $|Z| \cap K_{1}=\emptyset$ where $K_{1}=\left(w+\varepsilon \overline{E^{p}}\right) \times\left(\overline{E^{n-p}} \backslash\left(U_{1} \cup \ldots \cup U_{s}\right)\right)$.

Choose $\widetilde{z_{j}} \in D \cap\left(\left(w+\varepsilon E^{p}\right) \times U_{j}\right)$ for $j=1, \ldots, s$. Then

$$
\mu(Z)=\sum_{j=1}^{s} \operatorname{deg}\left(\{w\} \times U_{j}\right) \wedge Z=\sum_{j=1}^{s} \operatorname{deg}\left(\left\{\pi\left(\widetilde{z_{j}}\right)\right\} \times U_{j}\right) \wedge Z .
$$

For sufficiently large $\nu$ we have $\left|Z^{\nu}\right| \subset \Omega \backslash\left(K \cup K_{1}\right)$, and so

$$
\sum_{j=1}^{s} \operatorname{deg}\left(\left\{\pi\left(\widetilde{z_{j}}\right)\right\} \times U_{j}\right) \wedge Z=\sum_{j=1}^{s} \operatorname{deg}\left(\left\{\pi\left(\widetilde{z_{j}}\right)\right\} \times U_{j}\right) \wedge Z^{\nu}=\mu\left(Z^{\nu}\right) .
$$

Then $Z^{\nu} \in U(A, K)$ for sufficiently large $\nu$ and condition 3 follows.

$3 \Rightarrow 1$. Fix $a=\left(a_{1}, \ldots, a_{n}\right), L, U$ as in 1. By Proposition 2.5.4 and (2) we can assume that $a=0, L=\mathbb{C}\left\{e_{p+1}, \ldots, e_{n}\right\}$ and $\overline{E^{n-p}} \subset U$.

There is $\varepsilon>0$ such that $|Z| \cap\left(\varepsilon \overline{E^{p}} \times \partial E^{n-p}\right)=\emptyset$ and $\varepsilon \overline{E^{p}} \times \overline{E^{n-p}} \subset \Omega$. Moreover,

$$
\left|Z^{\nu}\right| \cap\left(\left(\varepsilon \overline{E^{p}} \times \partial E^{n-p}\right) \cup\left(\{0\}^{p} \times\left(U \backslash E^{n-p}\right)\right)\right)=\emptyset
$$

and

$$
\mu\left(f^{*}\left(Z^{\nu}\right)\right)=\mu\left(f^{*}(Z)\right),
$$

where $f=\left(\varepsilon \operatorname{id}_{\mathbb{C}^{p}}, \operatorname{id}_{\mathbb{C}^{n-p}}\right)$ and $\nu$ is large enough.

The set $\left|Z^{\nu}\right| \cap U$ is compact and non-empty, hence $\operatorname{dim}\left(\left|Z^{\nu}\right| \cap U\right)=0$. By (1),

$$
\operatorname{deg}_{U}\left(Z^{\nu} \wedge L\right)=\operatorname{deg}_{U}(Z \wedge L)
$$

Re mark. Condition 2 resembles the one given in [Ch, $\S 12.2]$. The following example shows the slight difference between them.

ExAmple 2.8. $\Omega=\mathbb{C}^{2}, Z^{\nu}=(\{1 / \nu\} \times \mathbb{C})+(\{1-1 / \nu\} \times \mathbb{C}), Z=$ $(\{0\} \times \mathbb{C})+(\{1\} \times \mathbb{C}), \widetilde{Z}=(\{0\} \times \mathbb{C})+2(\{1\} \times \mathbb{C})$. One can see that $Z^{\nu} \rightarrow Z$ and $Z^{\nu} \rightarrow \widetilde{Z}$ in the sense of [Ch, $\left.\S 12.2\right]$. The definition in [Ch, §12.2] seems to be erroneous, for $\left[Z^{\nu}\right]$ does not converge to $[\widetilde{Z}]$ as a sequence of currents. Neither does it converge to $\widetilde{Z}$ in the topology of $p$-chains.

Let us define:

- $\mathcal{A}_{\mathbb{Q}}(\Omega)=\left\{f \in \mathcal{A}(\Omega): f(0), f\left(e_{1}\right), \ldots, f\left(e_{n}\right) \in(\mathbb{Q}+i \mathbb{Q})^{n}\right\}$,

- $\widetilde{\mathcal{K}}=\left\{\left[q_{1}, q_{2}\right] \times \ldots \times\left[q_{4 n-1}, q_{4 n}\right]: q_{1}, \ldots, q_{4 n} \in \mathbb{Q}\right\}$,

- $\mathcal{K}=\{\cup \mathcal{B}: \mathcal{B} \subset \widetilde{\mathcal{K}}, \mathcal{B}$ is finite $\}$,

- $\mathcal{U}_{\mathbb{Q}}(\Omega)=\left\{U(A, K): A \subset \mathcal{A}_{\mathbb{Q}}(\Omega) \times \mathbb{Z}, A\right.$ is finite, $\left.K \in \widetilde{\mathcal{K}}, K \subset \Omega\right\}$,

- $E\left(r_{1}, r_{2}\right)=r_{1} E^{p} \times r_{2} E^{n-p}$ for $r_{1}, r_{2}>0$.

TheOREM 2.9. $\mathcal{U}_{\mathbb{Q}}(\Omega)$ is a base for the topology of $p$-chains in $\Omega$. 
Proof. The assertion is obvious for $p=0$. Suppose that $p>0$ and let $Z=\sum_{j \in J} k_{j} Z_{j} \in U(A, K)$. We can assume $A=\left\{\left(f_{1}, c_{1}\right)\right\}$ (see the proof of Proposition 2.7). Then there are $\widetilde{K} \in \mathcal{K}$ and $\varepsilon>0$ satisfying

$$
\begin{gathered}
\widetilde{K} \cap|Z|=\emptyset, \quad K \subset \widetilde{K} \subset \Omega, \quad f_{1}(E(1+\varepsilon, 1+\varepsilon)) \subset \Omega, \\
f_{1}(E(1+\varepsilon, 1+\varepsilon) \backslash \overline{E(1+\varepsilon, 1-\varepsilon)}) \subset \widetilde{K} .
\end{gathered}
$$

Fix $0<r<1$. By a simple computation there is a neighborhood $U \subset \mathcal{A}(\Omega)$ of $f_{1}$ in the Banach space of affine mappings $\mathbb{C}^{n} \rightarrow \mathbb{C}^{n}$ such that each $f \in U$ satisfies the following conditions:

(5) $\quad f(E(1+\varepsilon / 2,1+\varepsilon / 2) \backslash \overline{E(1+\varepsilon / 2,1-\varepsilon / 2)}$

$$
\subset f_{1}(E(1+\varepsilon, 1+\varepsilon) \backslash \overline{E(1+\varepsilon, 1-\varepsilon)}),
$$

(6) $f_{1}\left(\{0\}^{p} \times E^{n-p}\right) \subset f(E(r / 2,1+\varepsilon / 2)) \subset f_{1}(E(r, 1+\varepsilon))$,

(7) $\quad\left(f_{1}^{-1} \circ f\right)\left(\{0\}^{p} \times E^{n-p}\right) \cap\left(E^{n} \backslash f_{1}^{-1}(\widetilde{K})\right)$

$$
=\left(f_{1}^{-1} \circ f\right)\left(\{0\}^{p} \times \mathbb{C}^{n-p}\right) \cap\left(E^{n} \backslash f_{1}^{-1}(\widetilde{K})\right),
$$

(8) $\left(f_{1}^{-1} \circ f\right)\left(\{0\}^{p} \times \mathbb{C}^{n-p}\right) \cap E^{n} \subset E(r, 1)$,

(9) $\pi_{(p+1, \ldots, n)} \mid\left(f_{1}^{-1} \circ f\right)\left(\{0\}^{p} \times \mathbb{C}^{n-p}\right)$ is a bijection.

Let $f \in U$ and $W=\sum l_{j} W_{j} \in U\left(\left\{\left(f, c_{1}\right)\right\}, \widetilde{K}\right)$. Inclusions (4) and (5) give

$$
\left(f_{1}^{-1}(|W|) \cup f^{-1}(|W|)\right) \cap\left(\overline{E^{p}} \times \partial E^{n-p}\right)=\emptyset .
$$

If $f_{1}^{-1}\left(W_{j}\right) \cap E^{n}=\emptyset$ then by $(4), f_{1}^{-1}\left(W_{j}\right) \cap E(1,1+\varepsilon)=\emptyset$. So, according to $(6), f^{-1}\left(W_{j}\right) \cap E(r / 2,1+\varepsilon / 2)=\emptyset$. Thus, by Remmert's theorem we have $f^{-1}\left(W_{j}\right) \cap E^{n}=\emptyset$. Similarly $f^{-1}\left(W_{j}\right) \cap E^{n}=\emptyset \Rightarrow f_{1}^{-1}\left(W_{j}\right) \cap E^{n}=\emptyset$, which gives $\left\{j: f^{-1}\left(W_{j}\right) \cap E^{n} \neq \emptyset\right\}=\left\{j: f_{1}^{-1}\left(W_{j}\right) \cap E^{n} \neq \emptyset\right\}$.

By (1),

$$
\mu\left(f_{1}^{*}\left(W_{j}\right)\right)=\operatorname{deg}\left(f_{1}^{-1}\left(W_{j}\right) \wedge\left(\{0\}^{p} \times E^{n-p}\right)\right) .
$$

By [Wi, Theorem 9.1] and (8), (9),

$\operatorname{deg}_{E^{n}}\left(f_{1}^{-1}\left(W_{j}\right) \wedge\left(\{0\}^{p} \times E^{n-p}\right)\right)=\operatorname{deg}_{E^{n}}\left(f_{1}^{-1}\left(W_{j}\right) \wedge\left(f_{1}^{-1} \circ f\right)\left(\{0\}^{p} \times \mathbb{C}^{n-p}\right)\right)$.

From (7),

$$
\begin{aligned}
\operatorname{deg}_{E^{n}}\left(f_{1}^{-1}\left(W_{j}\right) \wedge\left(f_{1}^{-1} \circ f\right)\left(\{0\}^{p} \times \mathbb{C}^{n-p}\right)\right) & \\
& =\operatorname{deg}_{E^{n}}\left(f_{1}^{-1}\left(W_{j}\right) \wedge\left(f_{1}^{-1} \circ f\right)\left(\{0\}^{p} \times E^{n-p}\right)\right) .
\end{aligned}
$$

By (4) and (6),

$$
\begin{array}{r}
\operatorname{deg}_{E^{n}}\left(f_{1}^{-1}\left(W_{j}\right) \wedge\left(f_{1}^{-1} \circ f\right)\left(\{0\}^{p} \times E^{n-p}\right)\right) \\
=\operatorname{deg}\left(f_{1}^{-1}\left(W_{j}\right) \wedge\left(f_{1}^{-1} \circ f\right)\left(\{0\}^{p} \times E^{n-p}\right)\right), \\
\operatorname{deg}\left(f_{1}^{-1}\left(W_{j}\right) \wedge\left(f_{1}^{-1} \circ f\right)\left(\{0\}^{p} \times E^{n-p}\right)\right)=\operatorname{deg}\left(W_{j} \wedge f\left(\{0\}^{p} \times E^{n-p}\right)\right) \\
=\operatorname{deg}\left(f^{-1}\left(W_{j}\right) \wedge\left(\{0\}^{p} \times E^{n-p}\right)\right)=\mu\left(f^{*}\left(W_{j}\right)\right) .
\end{array}
$$


We have obtained $Z \in U\left(\left\{\left(f, c_{1}\right)\right\}, \widetilde{K}\right) \subset U\left(\left\{\left(f_{1}, c_{1}\right)\right\}, K\right)$. Density of $\mathcal{A}_{\mathbb{Q}}(\Omega)$ in $\mathcal{A}(\Omega)$ ends the proof.

3. Metric on $\mathcal{G}^{p}(\Omega)$. Let $Z \in \mathcal{G}^{p}(\Omega)$. For each compact subset $K$ of $\Omega$ we fix $0<d_{K}<\min \{1, \operatorname{dist}(K, \partial \Omega)\}$ and define $H(K)=\bigcup_{x \in K} B\left(x, d_{K}\right)$.

DeFinition 3.1.

$$
d(Z, K)= \begin{cases}\operatorname{dist}(|Z| \cap H(K), K) & \text { if }|Z| \cap H(K) \neq \emptyset, \\ d_{K} & \text { if }|Z| \cap H(K)=\emptyset .\end{cases}
$$

LeMma 3.2. $d(\cdot, K)$ is continuous.

Proof. Let $Z^{\nu} \rightarrow Z$ and $d(Z, K)>0$. Fix $\widetilde{d}<d(Z, K)$. Then we have $\left|Z^{\nu}\right| \cap \overline{\bigcup_{x \in K} B(x, \widetilde{d})}=\emptyset$ for almost all $\nu$. We obtain $\liminf _{\nu \rightarrow \infty} d\left(Z^{\nu}, K\right) \geq$ $d(Z, K)$. If $|Z| \cap H(K)=\emptyset$ then $d(Z, K)=d_{K}$ and the lemma follows.

If $|Z| \cap H(K) \neq \emptyset$ then $\operatorname{dist}(|Z| \cap H(K), K)=|z-y|$ where $y \in K$, $z \in|Z| \cap \overline{H(K)}$. By Rückert's lemma there is a sequence $\left\{z_{\nu}\right\}, z_{\nu} \in\left|Z^{\nu}\right|$, $z_{\nu} \rightarrow z$, which gives

$$
\limsup _{\nu \rightarrow \infty} d\left(Z^{\nu}, K\right) \leq d(Z, K) .
$$

By the same argument the previous inequality holds when $d(Z, K)=0$.

Let $l \in \mathbb{Z}$ and let $\overline{E^{n}} \subset \Omega$.

Definition 3.3. If $|Z| \cap\left(\overline{E^{p}} \times \partial E^{n-p}\right)=\emptyset,|Z| \cap E^{n} \neq \emptyset, \mu(Z)=l$ we define $m_{l}(Z)=d\left(Z, \overline{E^{p}} \times \partial E^{n-p}\right)$. We put $m_{l}(Z)=0$ otherwise.

LEMMA 3.4. $m_{l}$ is continuous.

Proof. Let $Z^{\nu} \rightarrow Z$. If $m_{l}(Z) \neq 0$ then $m_{l}\left(Z^{\nu}\right)=d\left(Z^{\nu}, \overline{E^{p}} \times \partial E^{n-p}\right)$ for sufficiently large $\nu$ and we can use Lemma 3.2. If $m_{l}(Z)=0$ and $|Z| \cap\left(\overline{E^{p}} \times \partial E^{n-p}\right) \neq \emptyset$ then $\left|m_{l}\left(Z^{\nu}\right)\right| \leq\left|d\left(Z^{\nu}, \overline{E^{p}} \times \partial E^{n-p}\right)\right| \rightarrow 0$. Suppose that $|Z| \cap\left(\overline{E^{p}} \times \partial E^{n-p}\right)=\emptyset$ and $|Z| \cap E^{n}=\emptyset$. By Remmert's theorem $\left|Z^{\nu}\right| \cap E^{n}=\emptyset$ for almost all $\nu$. If $m_{l}(Z)=0,|Z| \cap\left(\overline{E^{p}} \times \partial E^{n-p}\right)=\emptyset$ and $|Z| \cap E^{n} \neq \emptyset$, then

$$
\mu\left(Z^{\nu}\right)=\mu(Z) \neq l
$$

for sufficiently large $\nu$.

Set $\mathcal{P}(\Omega)=\left\{m_{l} \circ f^{*}: f \in \mathcal{A}_{\mathbb{Q}}(\Omega), l \in \mathbb{Z}\right\}$, and observe that we have

$$
\left\{\prod_{h \in J} h \cdot d(\cdot, K): J \subset \mathcal{P}(\Omega), J \text { is finite, } K \in \mathcal{K}\right\},
$$

a countable family of continuous functions. Let $\left\{G_{j}\right\}$ denote a sequence of all its elements. 
Definition 3.5. Let $X, Z \in \mathcal{G}^{p}(\Omega)$. We define

$$
\varrho(X, Z)=\sum_{j=0}^{\infty} \frac{1}{2^{j}}\left|G_{j}(X)-G_{j}(Z)\right| .
$$

THEOREM 3.6. $\varrho$ is a metric on $\mathcal{G}^{p}(\Omega)$. The topology induced by $\varrho$ coincides with the topology of $p$-chains.

Proof. It is sufficient to prove that the sequence $\left\{G_{j}\right\}$ gives an embedding of $\mathcal{G}^{p}(\Omega)$ in the Hilbert cube. According to [En, 2.3, Theorem 10] we need to prove that:

1. $\left\{G_{j}\right\}_{j \in \mathbb{N}}$ separates elements of $\mathcal{G}^{p}(\Omega)$,

2. $\left\{G_{j}\right\}_{j \in \mathbb{N}}$ separates elements of $\mathcal{G}^{p}(\Omega)$ from closed subsets of $\mathcal{G}^{p}(\Omega)$.

1) We can assume that $|Z| \neq \emptyset$. If $|X| \neq|Z|$ then there is $K \in \widetilde{K}$ satisfying $|X| \cap K=\emptyset,|Z| \cap K \neq \emptyset$. We obtain

$$
0=d(Z, K) \neq d(X, K) \text {. }
$$

Suppose $|X|=|Z|$. There is $z \in \operatorname{Reg}|X|$ satisfying $m(z, X) \neq m(z, Z)$. Fix $g \in \mathcal{A}_{\mathbb{Q}}(\Omega)$ such that $\mu\left(\pi \mid g^{-1}(|Z|)\right)=1$. Consequently,

$$
\left(m_{m(z, Z)} \circ g^{*}\right)(Z) \neq\left(m_{m(z, Z)} \circ g^{*}\right)(X)=0 .
$$

2) Let $X \in U\left(\left\{\left(f_{1}, c_{1}\right), \ldots,\left(f_{m}, c_{m}\right)\right\}, K\right) \subset \mathcal{G}^{p}(\Omega) \backslash C$, where $C$ is a closed subset of $\mathcal{G}^{p}(\Omega)$. Without loss of generality $U\left(\left\{\left(f_{1}, c_{1}\right), \ldots,\left(f_{m}, c_{m}\right)\right\}, K\right)$ $\in \mathcal{U}_{\mathbb{Q}}(\Omega)$.

If $|X| \neq \emptyset$ set $G_{n}=\prod_{j=1}^{m}\left(m_{c_{j}} \circ f_{j}^{*}\right) \cdot d(, K)$. If $|X|=\emptyset$ choose $G_{n}=$ $d(, \widetilde{K})$ where $\widetilde{K} \in \mathcal{K}$ and

$$
U(\widetilde{K}) \subset U\left(\left\{\left(f_{1}, c_{1}\right), \ldots,\left(f_{m}, c_{m}\right)\right\}, K\right) .
$$

In both cases we obtain $\left.G_{n}\right|_{C}=0, G_{n}(X) \neq 0$.

Acknowledgements. I would like to express my sincere thanks to Piotr Tworzewski for many helpful conversations.

\section{References}

[Ch] E. M. Chirka, Complex Analytic Sets, Kluwer Acad. Publishers, 1989.

[Dr] R. N. Draper, Intersection theory in analytic geometry, Math. Ann. 180 (1969), 175-204.

[En] R. Engelking, General Topology, Heldermann, 1989.

[Gu] R. C. Gunning, Introduction to Holomorphic Functions of Several Complex Variables. Local Theory, Wadsworth \& Brooks/Cole, 1990.

[Ha] R. Harvey, Holomorphic chains and their boundaries, in: Proc. Sympos. Pure Math. 30, Part 1, Amer. Math. Soc., 1977, 309-382. 
[Ło] S. Łojasiewicz, Introduction to Complex Analytic Geometry, Birkhäuser, Basel, 1991.

[R] S. Rams, Bezout-type theorem for certain analytic sets, in preparation.

[Tw] P. Tworzewski, Intersection theory in complex analytic geometry, Ann. Polon. Math. 62 (1995), 177-191.

[TW] P. Tworzewski and T. Winiarski, Continuity of intersection of analytic sets, ibid. 42 (1983), 387-393.

[Wi] T. Winiarski, Continuity of total number of intersection, ibid. 47 (1986), 155178.

Institute of Mathematics Jagiellonian University

Reymonta 4

30-059 Kraków, Poland

E-mail: rams@im.uj.edu.pl

Reçu par la Rédaction le 3.4.1996

Révisé le 26.7.1996 